\title{
THE
}

$4-11-1988$

\section{Monte Carlo Calculation of Free Energy, Critical Point, and Surface Critical Behavior of Three-Dimensional Heisenberg Ferromagnets}

\author{
M. P. Nightingale \\ University of Rhode Island, nightingale@uri.edu
}

H. W.J. Blöte

Follow this and additional works at: https://digitalcommons.uri.edu/phys_facpubs

Terms of Use

All rights reserved under copyright.

\section{Citation/Publisher Attribution}

M. P. Nightingale and H. W. J. Blöte. (1988). "Monte Carlo calculation of free energy, critical point, and surface critical behavior of three-dimensional Heisenberg ferromagnets." Physical Review Letters, 60(15), 1562. Available at: http://dx.doi.org/10.1103/PhysRevLett.60.1562

This Article is brought to you for free and open access by the Physics at DigitalCommons@URI. It has been accepted for inclusion in Physics Faculty Publications by an authorized administrator of DigitalCommons@URI. For more information, please contact digitalcommons-group@uri.edu. 


\title{
Monte Carlo Calculation of Free Energy, Critical Point, and Surface Critical Behavior of Three-Dimensional Heisenberg Ferromagnets
}

\author{
M. P. Nightingale \\ Department of Physics, University of Rhode Island, Kingston, Rhode Island 02881 \\ and \\ H. W. J. Blöte \\ Laboratorium voor Technische Natuurkunde, 2600 GA Delft, The Netherlands
}

(Received 18 January 1988)

\begin{abstract}
A transfer-matrix Monte Carlo technique is developed to compute the free energy of threedimensional, classical Heisenberg ferromagnets. From the free energy of systems with periodic and antiperiodic boundary conditions, helicity moduli are calculated. From these the critical couplings for simple cubic (sc) and face-centered cubic lattices are estimated, by use of finite-size scaling. For the sc lattice, the critical dimension of the surface magnetization is estimated with standard Monte Carlo methods, yielding a result in excellent agreement with the $\epsilon$-expansion work of Diehl and Nüsser.
\end{abstract}

PACS numbers: $75.40 . \mathrm{Cx}, 05.70 . \mathrm{Fh}, 64.60 . \mathrm{Fr}, 75.40 . \mathrm{Mg}$

Transfer matrices are widely used in numerical studies of statistical mechanical systems in two dimensions with discrete microscopic degrees of freedom. ${ }^{1}$ In three dimensions, numerical calculation of eigenvalues of transfer matrices become intractable already for small systems: a $5 \times 5 \times \infty$ Ising model in $2+1$ dimensions seems to be the current upper limit. ${ }^{2}$ Systems with continuous degrees of freedom, with the exception of linear chains, are even more of a challenge.

Here, we combine Monte Carlo (MC) and transfermatrix techniques to tackle a three-dimensional Heisenberg model and directly calculate free energies in terms of the transfer-matrix eigenvalues for lattices up to $10 \times 10 \times \infty$. The method is a variant of the Green'sfunction MC method, ${ }^{3}$ of which there have been some preliminary applications to the three-dimensional Ising model. ${ }^{4}$

Consider a lattice in three dimensions with $N$ sites: $n_{z}$ layers of $m=n_{x} n_{y}$ sites each. We chose helical boundary conditions to obtain a single, sparse transfer matrix, and label the sites with one index $i=1, \ldots, N$. Sites $1, \ldots, m$ and $N-m+1, \ldots, N$ form the bottom and top surfaces. For a general lattice, each nearest-neighbor bond features precisely once in the list $\left(i, i-d_{a}\right)$, $i=1, \ldots, N$, and $\alpha=1, \ldots, c$, up to end effects. The $c$ displacements $d_{a}$ define the lattice: $c=3$ with $d_{1}=1$, $d_{2}=n_{x}$, and $d_{3}=m$ yields the simple cubic (sc) lattice; for the face-centered cubic (fcc) lattice, add $d_{4}=m-1$, $d_{5}=m-n_{x}$, and $d_{6}=m-n_{x}-1$. At each site $i$ there is a spin, a three-component unit vector $\mathbf{s}_{i}$. The reduced Hamiltonian is

$$
-\beta \mathcal{H}=\sum_{i=1}^{N} \sum_{\alpha=1}^{c} \mathbf{s}_{i}^{\prime} \cdot \mathbf{s}_{i}^{\prime}-d_{\alpha},
$$

where $\mathbf{s}_{i}^{\prime}=\mathbf{s}_{i} \sqrt{ } K$ for coupling constant $K \quad\left(\mathbf{s}_{i}^{\prime}=0\right.$ for $i<0$ ). Integration of the Boltzmann weights over all $\mathbf{s}_{i}$ with $i \leqq N-m$ gives a partition function:

$$
Z_{N}\left(S_{N}^{N-m+1}\right)=\int \cdots \int d S_{1}^{N-m} e^{-\beta \mathcal{H}},
$$

where $S_{i}^{j}=\left(\mathbf{s}_{i}, \ldots, \mathbf{s}_{j}\right)$. With general $m$-uples of spins $U=\left(\mathbf{u}_{i}, \ldots, \mathbf{u}_{m}\right)$ and $V$, we define a transfer matrix $\mathbf{T}$ that adds one site to the lattice:

$$
\mathrm{T}(U \mid V)=\exp \left(\mathbf{u}_{1} \cdot \sum_{\alpha} \mathbf{v}_{d_{\alpha}}\right) \prod_{i=2}^{m} \delta\left(\mathbf{u}_{i}, \mathbf{v}_{i-1}\right),
$$

where the $\delta$ functions are normalized such that

$$
Z_{N+1}(U)=\int \cdots \int \mathrm{T}(U \mid V) Z_{N}(V) d V .
$$

As $N \rightarrow \infty$, the dimensionless free energy $(f)$ per site is given in terms of the dominant eigenvalue $\left(\lambda_{0}\right)$ of $\mathbf{T}$ by $f=-\ln \lambda_{0}$.

To implement the power method to obtain this eigenvalue, the matrix multiplication in Eq. (3) is represented by a random process, the so-called transfer-matrix MC method. Introduce a sequence of random walkers $R_{t}$ $=\left(S_{l}, w_{\imath}\right), \quad l=1, \ldots, r: \quad S_{l}=\left(\mathbf{s}_{1}^{1}, \ldots, \mathbf{s}_{m}^{l}\right)$ represents a layer configuration of statistical weight $w_{l} \geqq 0$. We maintain $r$ within a few percent of a target $r_{0}$; the weights are kept in the range $b_{l}<w_{l}<b_{u}$, with $b_{l} \approx \frac{1}{2}$ and $b_{u}=2$. Rewrite $\mathrm{T}\left(S^{\prime} \mid S\right)=P\left(S^{\prime} \mid S\right) D(S)$, with a normalization $D(S)$ independent of $S^{\prime}$, such that $P\left(S^{\prime} \mid S\right)$ is a probability density for a transition from $S$ to $S^{\prime}$. An MC run consists of sweeps $t=1, \ldots, M$ over all random walkers. At time $t$ there are two steps. Affixing primes to variables at time $t+1$, we define step (1): For $\imath=1, \ldots, r$ change $R_{l}$ to $R_{l}^{\prime}=\left(S_{l}^{\prime}, w_{l}^{\prime}\right)$ according to $P\left(S_{\imath}^{\prime} \mid S_{\imath}\right)$, with $w_{\imath}^{\prime}=D\left(S_{\imath}\right) w_{l} / c^{\prime}$. With $\hat{\lambda}_{0}$ a running estimate of $\lambda_{0}$, choose $c^{\prime}=\hat{\lambda}_{0} r / r_{0}$ to maintain $r$ close to $r_{0}$ in step (2): From the $R_{t}^{\prime}$ construct a new sequence 
using each walker precisely once: (a) If $w_{l}^{\prime}>b_{u}$, add two random walkers $\left(S_{\imath}^{\prime}, \frac{1}{2} w_{\imath}^{\prime}\right)$ to the new sequence; (b) form pairs $\left(S_{l}^{\prime}, w_{l}^{\prime}\right)$ and $\left(S_{\kappa}^{\prime}, w_{\kappa}^{\prime}\right)$ with $w_{l}^{\prime}<b_{l}$ and $w_{\kappa}^{\prime}<b_{l}$, and add $\left(S_{\lambda}^{\prime}, w_{\imath}^{\prime}+w_{\kappa}^{\prime}\right)$, where $S_{\lambda}^{\prime}=S_{\imath}^{\prime}$ or $S_{\lambda}^{\prime}=S_{\kappa}^{\prime}$ with relative probabilities $w_{l}^{\prime}$ and $w_{k}^{\prime}$; (c) if $b_{l}<w_{l}^{\prime}<b_{u}$, or if $R_{l}$ is left unpaired in step (b) add $R_{l}^{\prime}$. The walkers represent a vector $\Phi$ with components

$$
\boldsymbol{\Phi}(U)=\sum_{i=1}^{r} w_{l} \delta\left(\mathbf{s}_{1}^{l}, \mathbf{u}_{1}\right) \cdots \delta\left(\mathbf{s}_{m}^{l}, \mathbf{u}_{m}\right) .
$$

Denote the vector realized in sweep $t$ by $\boldsymbol{\Phi}_{t}(U)$. The crux of the method is that

$$
\left\langle\prod_{b=1}^{\tau} c_{t+b} \boldsymbol{\Phi}_{t+\tau}(U)\right\rangle=\mathbf{T}^{\tau} \boldsymbol{\Phi}_{t}(U),
$$

where the angle brackets denote the average over all processes starting from the vector $\boldsymbol{\Phi}_{t}(U)$. An estimator ${ }^{5}$ of the dominant eigenvector $\boldsymbol{\Psi}_{0}$ of the transfer matrix is

$$
\mathbf{\Psi}_{0}(U, \tau)=\frac{1}{M} \sum_{t=1}^{M}\left(\prod_{b=0}^{\tau} c_{t-b} \boldsymbol{\Phi}_{t}(U)\right)
$$

This is the iterate of order $\tau$ in the power method, and as such it has a bias for any $\tau<\infty$; its variance increases with $\tau$. As a compromise we chose the largest $\tau$ with a statistically significant nonzero estimate of the autocorrelation at lag $\tau$ of $c_{t}$. The dominant eigenvalue of the transfer matrix is given by $\lambda_{0} \approx W_{0}(\tau+1) / W_{0}(\tau)$, where $W_{0}$ is the integral of $\boldsymbol{\Psi}_{0}(U, \tau)$ over $U$. As $\boldsymbol{\Psi}_{0}(U)$ for $N \rightarrow \infty$ is the probability density of a surface configuration $U$, the multiplication of Eq. (6) through by a spin function and integration over $U$ yields surface correlations.

We applied the same method to systems with antiperiodic boundary conditions: Each of the $n_{z}$ planes had two seams related by translations by a vector between sites 0 and $n_{x} n_{y}$. One seam was in the $y$ direction, the other in the $x$ direction, except for a single step in the $y$ direction forced by the helical boundary conditions. Along bonds across the seam the coupling was $-K$, instead of $K$.

The critical coupling $K_{c}$ was obtained as follows. Denote the dimensionless free energies per site of the periodic and antiperiodic systems by $f_{+}$and $f_{-}$; write $\Delta=K_{c}-K$. For $n_{x}=n_{y}=n$ and small $|\Delta|$ one has the scaling relation

$$
n^{d}\left(f_{+}-f_{-}\right)=H\left(n^{y_{T}} \Delta\right) \approx H_{0}+H_{1} n^{y_{T}} \Delta
$$

in $d=3$ dimensions ${ }^{6} ; H$ is the helicity scaling function, and the correlation length diverges as $\Delta^{-v}$, where $y_{T}=v^{-1}$. With $K_{c}, H_{0}$, and $H_{1}$ as parameters, we made least-squares fits to data for several $K$ and $n$ values. In most runs the target number $r_{0}$ was 2500 . With a number of sweeps that added roughly 5000 layers, this amounts to $12.5 \times 10^{6}$ flips per spin in total.

Figure 1 shows $H$ vs $n$ on an $n^{y_{T}}$ scale for various $K$,

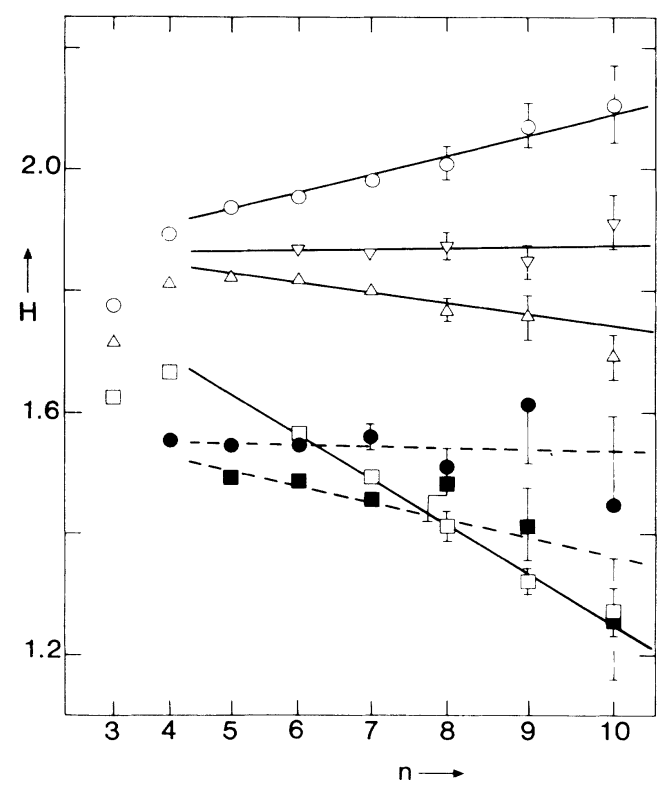

FIG. 1. Helicity modulus scaling function vs $n^{1.418}$ for various coupling strengths $K$. Open symbols represent data for the sc lattice: $K=0.695$ (circles), 0.6922 (inverted triangles), 0.6904 (triangles), and 0.68236 (squares); filled symbols are fcc data: $K=0.316$ (circles) and 0.31489 (squares). For $n=10$, two squares almost coincide; the fcc datum point has the longer error bars. Solid (sc) and dashed (fcc) lines illustrate linear behavior near criticality for $n>5$.

for both the sc and fcc lattices. To check for finite-size effects, we systematically increased the size of the smallest system included in the fits. The results (and standard errors) are as follows: For the sc lattice, $K_{c}=0.6922(2)$ and $0.6925(3)$, for sizes 5 and up and 6 and up, respectively; for the fcc lattice, $K_{c}=0.3162(3), 0.3160(2)$, and $0.3170(5)$, for sizes 4 and up, 5 and up, and 6 and up. In the fits we used ${ }^{7} y_{T}=1.418$. The $K_{c}$ for the sc case agrees well with results of Ritchie and Fisher ${ }^{8}$ and of Ferer $^{9}$ obtained from eight- and twelve-term series. Agreement is less satisfactory with a ten-term-series estimate of Ohno, Okabe, and Morita ${ }^{10}$ who find $K_{c}$ $\approx 0.68236$. For the fcc lattice the agreement with the result ${ }^{11} K_{c}=0.3149(2)$ is reasonable. The values of $K$ around $K_{c}$ were not chosen optimally to estimate the bulk thermal exponent, yet we have data for the sc lattice over a sufficiently wide range of couplings to obtain least-squares estimates: $y_{T}=1.406(55)$ and 1.396(78) for sizes 5 and up and 6 and up, respectively.

The transfer-matrix method was also used to calculate surface correlations for the Heisenberg system. Only for small systems was a variance obtained smaller than with a standard MC algorithm, ${ }^{12}$ and so only results of the latter will be discussed here. We used finite sc lattices with sites $(x, y, z)$, with $x, y$, and $z$ ranging from 1 to $n_{x}=n_{y}=n$ and $n_{z}=2 n$, respectively, free boundaries at $z=1$ and $z=n_{z}$, and helical or periodic boundary condi- 
tions in $x$ and $y$ directions. Up to boundary effects, the Hamiltonian was that of Eq. (1). Also, couplings with and within the surface were redefined via $\mathbf{s}_{i}^{\prime}=\mathbf{s}_{i} \sqrt{ } K_{s}$, where $K_{s}=\epsilon K$ with enhancement factor $\epsilon$. We calculated the surface susceptibility

$$
\chi_{11}=n^{-2}\left\langle\sum \mathbf{s}_{x, y, z} \cdot \mathbf{s}_{x^{\prime}, y^{\prime}, z}\right\rangle,
$$

summing over all sites $(x, y, z)$ and $\left(x^{\prime}, y^{\prime}, z\right)$ on one surface; the angle brackets denote the thermal average. We also calculated the surface correlation $g_{n}$ halfway across the system:

$$
\begin{aligned}
g_{n}=n^{-2} \sum_{x} \sum_{y}\left\langle\mathbf { s } _ { x , y , z } \cdot \left(\mathbf{s}_{x+n / 2, y, z}\right.\right. & +\mathbf{s}_{x, y+n / 2, z} \\
& \left.\left.+\mathbf{s}_{x+n / 2, y+n / 2, z}\right)\right\rangle,
\end{aligned}
$$

identifying sites $(x, y, z)$ and $\left(x^{\prime}, y^{\prime}, z\right)$, if $\left|x-x^{\prime}\right|=n$, or $\left|y-y^{\prime}\right|=n$. With $\chi_{11}$ and $g_{n}$ we estimated the surface critical exponent $y_{H_{1}}$, which, e.g., yields the surface susceptibility exponent with $\gamma_{11}=\left(d^{\prime}-2 y_{H_{1}}\right) / y_{T}$, where $d^{\prime}=2$.

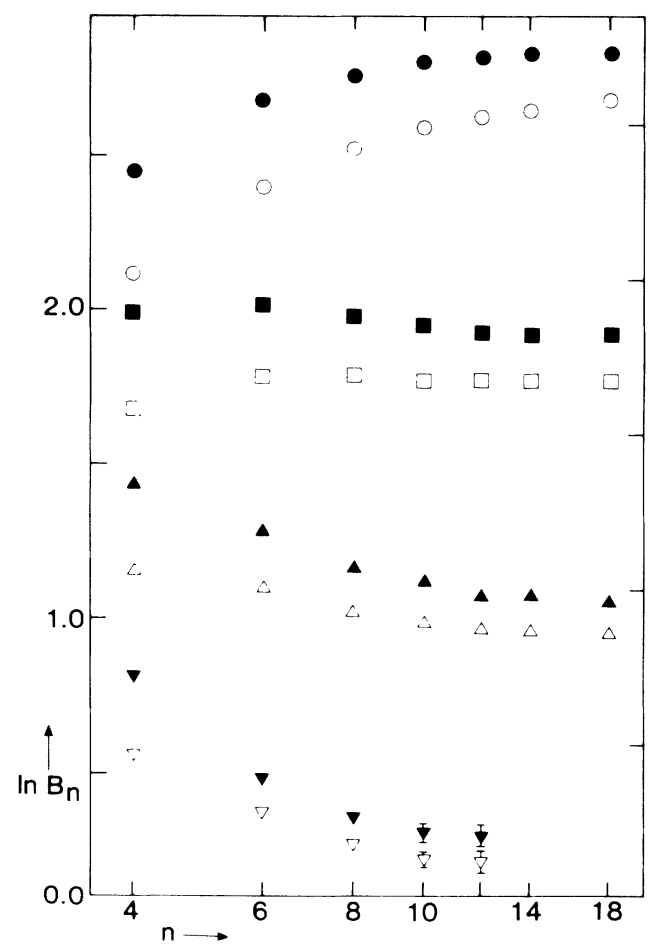

FIG. 2. Scaling behavior of surface correlation function $g_{n}$ : $\ln B_{n}$ vs $n$ on a $\ln n$ scale for several values of the surface enhancement $\epsilon$, where $B_{n}=g_{n} \exp \left[\left(4-2 y_{H_{1}}\right) \ln n\right]$, with $y_{H_{1}}$ $=0.8$ from our numerical analysis. According to scaling, $B_{n}$ is finite and nonzero for $n \rightarrow \infty$. Filled and open symbols are for cylindrical and helical boundary conditions, respectively: $\epsilon=1.0$ (circles), 0.83 (squares), 0.66 (triangles), and 0.5 (inverted triangles). To avoid overlap, data points for cylindrical systems are shifted upward by 0.1 (see tick marks on the right-hand side). Error bars are shown where they exceed the symbol size.
The MC estimates were obtained from one or several runs of $2 \times 10^{5}$ flips per spin. To obtain a vectorizable algorithm, spins were flipped sequentially on sublattices, such that nearest neighbors were on different sublattices. For periodic lattices of even size this is simple: They are bipartite. Helical systems had $p$ sublattices $L_{i}: L_{i}$ consists of sites $i+k p(k=0,1, \ldots)$, with $p$ the smallest integer relatively prime to $n$ and $n^{2}$.

The MC data (see Figs. 2 and 3) were analyzed with finite-size scaling. The $\gamma_{11}$ were fitted ${ }^{13}$ with $\chi_{11}(n)$ $\approx \chi_{11}(\infty)+A n^{g}$, where $g=d^{\prime}-2 y_{H_{1}}$. The $g_{n}$ have strong corrections to scaling and were fitted with $g_{n}$ $\approx(B+C / n) n^{h}$, where $h=2\left(y_{H_{1}}-d^{\prime}\right)$. A renormalization-group argument suggests the origin and sign of this correction. Simply assume that the surface fixed point ${ }^{14}$ is characterized by a single, nearest-neighbor interaction $K_{s}^{*}$. At bulk criticality $K_{s}$ will tend towards $K_{s}^{*}$ under renormalization. As the number of renormalizations required to calculate correlations grows with distance, they will decay with an effective exponent $y_{H_{1}}$ which decreases with distance for $K_{s}>K_{s}^{*}$ and vice versa. Scaling ${ }^{15}$ and $\epsilon$-expansion ${ }^{16}$ arguments suggest the naive

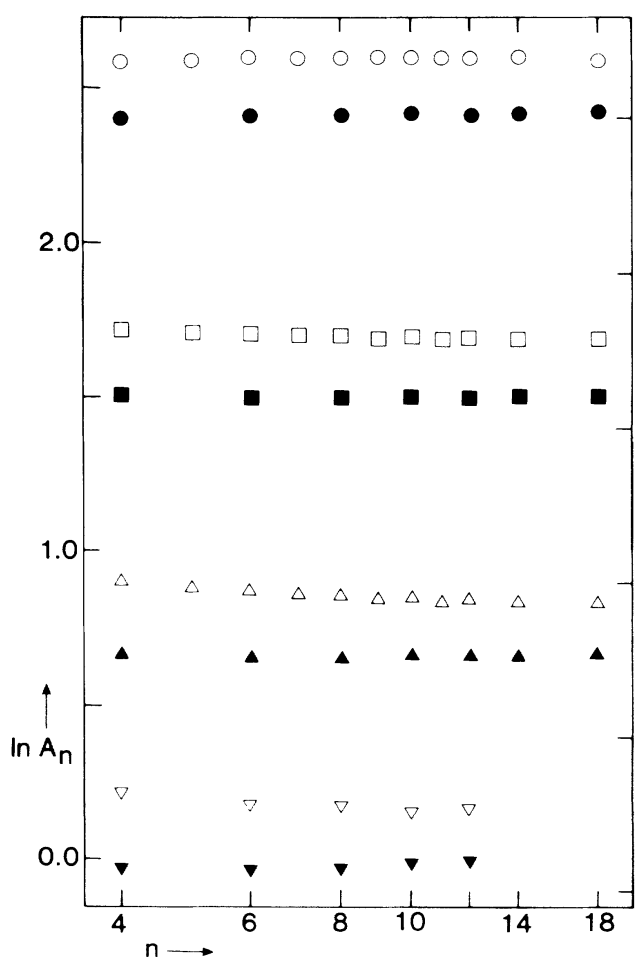

FIG. 3. Scaling behavior of surface susceptibility $\chi_{11}: \ln A_{n}$ vs $n$ on a $\ln n$ scale for several values of the surface enhancement $\epsilon$, where $A_{n}=\left[\chi_{11}(\infty)-\chi_{11}(n)\right] \exp \left[\left(2 y_{H_{1}}-2\right) \ln n\right]$, with $y_{H_{1}}=0.8$ and $\chi_{11}(\infty)=11.25,6.05,3.65$, and 2.50 for $\epsilon=1.0$, $0.83,0.66$, and 0.5 from our numerical analysis. For the key to the symbols see Fig. 2. To avoid overlap, data points for cylindrical systems are shifted upward by 0.1 (see tick marks on the right-hand side). The statistical errors in the data do not exceed the size of the symbols. 
TABLE I. Estimates of surface critical exponent $y_{H_{1}}$ obtained from least-squares fits to surface susceptibility and correlation data for both helix and cylinder boundary conditions. Results are labeled by $n$, the smallest system size included in the various fits.

\begin{tabular}{rcccc}
\hline \hline & \multicolumn{2}{c}{$\chi_{11}$} & & \multicolumn{2}{c}{$g_{n}$} \\
$n$ & Helix & Cylinder & Helix & Cylinder \\
\hline 4 & $0.804(11)$ & $0.778(07)$ & $0.696(11)$ & $0.685(12)$ \\
5 & $0.809(10)$ & & & \\
6 & $0.792(11)$ & $0.806(11)$ & $0.753(14)$ & $0.770(18)$ \\
7 & $0.793(17)$ & & & \\
8 & $0.774(21)$ & $0.791(24)$ & $0.785(23)$ & $0.784(32)$ \\
9 & $0.801(36)$ & & & \\
10 & $0.776(50)$ & $0.699(49)$ & $0.823(36)$ & $0.821(72)$ \\
\hline \hline
\end{tabular}

value -1 for the correction-to-scaling exponent. The analysis of $\chi_{11}$ required no such correction. Estimates of $y_{H_{1}}$ were obtained from $\chi_{11}$ and $g_{n}$ for several enhancements $\epsilon$, both for cylinders and helices. Table I summarizes the results. We attribute the deviations at the top and bottom of Table I to finite size and too small a range of system sizes, respectively, and find $y_{H}=0.80 \pm 0.03$. This confirms the $\epsilon$-expansion value ${ }^{17} \quad y_{H_{1}}=0.809$ \pm 0.014 , the series estimate ${ }^{10} y_{H_{1}}=0.85 \pm 0.06$, a previous $\mathrm{MC}$ result ${ }^{18} y_{H_{1}}=0.87 \pm 0.14$, and the experimental value ${ }^{19} y_{H_{1}}=0.83 \pm 0.06$.

The authors thank Professor M. Ferer for his estimate of the critical temperature of the sc system, and Dr. R. G. Caflisch, Professor K. K. Mon, and Professor J. J. Rehr for discussions. This work, done in part at the Aspen Center for Physics, was funded by the National Science Foundation under Contract No. DMR-87-04730, and by NATO under Grant No. 198/84. It was conducted (with special thanks to Marcia Pottle) with use of the Cornell National Superconducting Facility, which is funded in part by the National Science Foundation, New
York State, and the IBM Corporation.

${ }^{1}$ For a review see, e.g., M. P. Nightingale, J. Appl. Phys. 53, 7932 (1982).

${ }^{2}$ C. J. Hamer and C. H. J. Johnson, J. Phys. A 19, 423 (1986)

${ }^{3}$ D. M. Ceperley and M. H. Kalos, in Monte Carlo Methods in Statistical Physics, edited by K. Binder (Springer-Verlag, Berlin, 1979).

${ }^{4} \mathrm{~J}$. Kuti, in Monte Carlo Methods in Quantum Mechanical Problems, edited by M. H. Kalos, NATO Advanced Study Institute, Series B, Vol. 125 (Plenum, New York, 1984).

${ }^{5}$ J. H. Hetherington, Phys. Rev. A 30, 2713 (1984); M. P. Nightingale and H. W. J. Blöte, Phys. Rev. B 33, 659 (1986).

${ }^{6}$ M. P. M. den Nijs, M. P. Nightingale, and M. Schick, Phys. Rev. B 26, 2490 (1982).

${ }^{7}$ J. C. le Guillou and J. Zinn-Justin, Phys. Rev. B 21, 3976 (1980), and J. Phys. (Paris) Lett. 46, L137 (1985).

${ }^{8}$ D. S. Ritchie and M. E. Fisher, Phys. Rev. B 7, 480 (1973).

${ }^{9} \mathrm{M}$. Ferer, unpublished.

${ }^{10}$ K. Ohno, Y. Okabe, and A. Morita, Prog. Theor. Phys. 71, 714 (1984).

${ }^{11}$ M. Ferer and A. Hamid-Aidinejad, Phys. Rev. B 34, 6481 (1986).

${ }^{12}$ Th. T. A. Paauw, A. Compagner, D. Bedeaux, Physica (Amsterdam) 79A, 1 (1975).

${ }^{13}$ See, for an analogous finite-size expression, $M . P$. Nightingale and H. W. J. Blöte, J. Phys. A 15, L33 (1982).

${ }^{14}$ N. M. Svrackič and M. Wortis, Phys. Rev. B 15, 396 (1977).

${ }^{15}$ T. W. Burkhardt and J. L. Cardy, J. Phys. A 20, L233 (1987)

${ }^{16}$ H. W. Diehl and S. Dietrich, Z. Phys. B 50, 117 (1983).

${ }^{17}$ H. W. Diehl and A. Nüsser, Phys. Rev. Lett. 56, 2834 (1986).

${ }^{18}$ K. Binder and P. C. Hohenberg, Phys. Rev. B 9, 2194 (1974).

${ }^{19}$ S. F. Alvarado, M. Campagna, and H. Hopster, Phys. Rev. Lett. 48, 51 (1982); S. F. Alvarado, M. Campagna, F. Ciccacci, and H. Hopster, J. Appl. Phys. 11, 7920 (1982). 